\title{
MicroRNA-15b-5p exerts its tumor repressive role via targeting GDI2: A novel insight into the pathogenesis of thyroid carcinoma
}

\author{
JIDONG ZOU $^{1}$, JIANTONG QIAN ${ }^{2}$, HAIYAN FU $^{3}$, FAWEN YIN ${ }^{1}$, WANJUN ZHAO ${ }^{1}$ and LIANG XU ${ }^{1}$ \\ ${ }^{1}$ Thyroid Diseases Department, Shandong Provincial ENT Hospital, Cheeloo College of Medicine, \\ Shandong University, Jinan, Shandong 250022; ${ }^{2}$ Otolaryngology Department, Traditional Chinese Medicine \\ Hospital of Juxian, Rizhao, Shandong 276599; ${ }^{3}$ Pathology Department, Shandong Provincial ENT Hospital, \\ Cheeloo College of Medicine, Shandong University, Jinan, Shandong 250022, P.R. China
}

Received January 17, 2020; Accepted June 12, 2020

DOI: $10.3892 / \mathrm{mmr} .2020 .11343$

\begin{abstract}
Thyroid carcinoma (THCA) is a malignant tumor of the endocrine system. Previous studies have revealed the vital roles of microRNAs (miRNAs/miRs) in THCA procession. The present study aimed to explore the effects of miR-15b-5p on the progression of THCA and its targeting mechanism. The data of THCA and healthy samples were firstly collected from starbase 2.0 and used to analyze the relationship of miR-15b-5p with THCA. Dual-luciferase assay was performed to detect the direct interaction between miR-15b-5p and the predicted target gene GDP dissociation inhibitor 2 (GDI2). The effects of miR-15b-5p and GDI2 on the overall survival of patients with THCA were analyzed using Kaplan-Meier analysis with $\log$ rank test. Cell Counting Kit-8 and Transwell assays were conducted to assess the impacts of miR-15b-5p and GDI2 on the proliferation and invasion of THCA cells. Reverse transcription-quantitative PCR and western blot analyses were performed to analyze the expression levels of the related miRNAs and proteins, respectively. miR-15b-5p was found to be downregulated both in THCA tissues and cells, and the low expression of miR-15b-5p was associated with the short overall survival time of patients. Moreover, the upregulation or downregulation of miR-15b-5p could inhibit or enhance the proliferation and invasion of THCA cells, respectively. miR-15b-5p reduced the protein expression levels of matrix metalloproteinase (MMP)2 and MMP9, which were related to cell invasion. Furthermore, GDI2, which was enhanced in THCA and related to the poor prognosis of patients with THCA, was identified as the target gene of miR-15b-5p and negatively regulated by miR-15b-5p. Additional experiments
\end{abstract}

Correspondence to: Dr Jidong Zou, Thyroid Diseases Department, Shandong Provincial ENT Hospital, Cheeloo College of Medicine, Shandong University, 4 Duanxing Xi Road, Huaiyin, Jinan, Shandong 250022, P.R. China

E-mail: entzjd@126.com

Key words: microRNA-15b-5p, thyroid carcinoma, GDP dissociation inhibitor 2, proliferation, migration demonstrated that GDI2 overexpression could significantly reduce the antitumor effect of miR-15b-5p and its inhibitory action on the expression levels of MMP2 and MMP9. Thus, the results indicated a potential tumor suppressive role of miR-15b-5p in THCA, which was mainly exerted by targeting GDI2 and modulating MMP2 and MMP9. These findings will increase the understanding on the pathogenesis of THCA and provide novel candidates for THCA therapy.

\section{Introduction}

Thyroid carcinoma (THCA) is a common type of malignant tumor of the endocrine system, representing $3.4 \%$ of all cancer types diagnosed annual $(1,2)$. The overall incidence of THCA is increasing in recent decades (3), with 567,000 incident cases of THCA reported in 2018 worldwide (4). Moreover, the morbidity of THCA in women is three times higher compared with men $(1,5)$, and despite the advancement for the treatment of THCA, the prognosis of patients remains poor (6). Therefore, it is important to provide novel insights into the gene regulatory circuits underlying THCA and identify new effective therapy targets.

MicroRNAs (miRNAs/miRs), a type of endogenous RNAs with a length of 18-22 nucleotides, have been reported to be vital regulators in various tumors (7). For example, miRNAs are involved in numerous processes of tumorigenesis such as cell proliferation, cell apoptosis, cell differentiation and development $(8,9)$. In addition, the expression of certain genes can be regulated by miRNAs via the binding between miRNAs and the 3'-untranslated region (3'-UTR) of the genes $(10,11)$. Previous studies have reported that miRNAs can not only serve as oncogenes, but also can exert a tumor suppressive role by regulating the expression of the target genes (12-14). As a mature miRNA, miR-15b-5p is spliced from the 5 '-end of pre-miR-15b, which is a member of miR-16 family $(15,16)$. Moreover, miR-15b-5p is upregulated in gastric cancer (15), liver cancer (17) and hepatocellular carcinoma (18-22), while it is lowly expressed in neuroblastoma (18) and prostate cancer (23). However, the biological functions and underlying mechanisms of miR-15b-5p in THCA are yet to be elucidated.

GDP dissociation inhibitor 2 (GDI2) belongs to a small family of chaperone proteins and is mainly expresses in 
hematopoietic, endothelial and epithelial cells $(24,25)$. Although the dysregulation of GDI2 has been revealed in numerous cancer types, including pancreatic carcinoma, ovarian cancer, gastric cancer and esophageal squamous cell carcinoma (26-30), the functions of GDI2 in cancer have been rarely reported. While Onda et al (31) demonstrated that GDI2 was upregulated in anaplastic thyroid cancer, the detailed effects of GDI2 and its regulatory mechanism in THCA remain unknown.

In the present study, the effects of miR-15b-5p on the prognosis of THCA patients, viability and invasion of THCA cells were identified. Moreover, the potential target gene of miR-15b-5p in THCA was verified by bioinformatics prediction, luciferase reporter assay and a series of rescue experiments. The results of the present study provide a novel insight in the pathological mechanism of THCA.

\section{Materials and methods}

Data collection and analysis. The data of tissue samples including 509 patients with THCA (age: $15-89$ years; 136 males and 373 females) and 58 healthy individuals were downloaded from the Cancer Genome Atlas project using starbase2.0 (http://starbase.sysu.edu.cn/index.php). The expressions of miRNAs or genes in different groups were analyzed using starbase2.0. Then, the patients of THCA were divided into high miR-15b-5p expression group (value $\geq 194.72$ ) and low miR-15b-5p expression group (value <194.72) according to the median expression of miR-15b-5p. The overall survival time of patients with THCA in two groups were analyzed using Kaplan-Meier analysis followed by log rank test.

Prediction of target gene for miR-15b-5p. The miRNAs target gene prediction websites TargetScan Release 7.2 (http://www.targetscan.org/vert_72/), miRanda 2010 Release (http://www.microrna.org/microrna/getGeneForm.do) and miRWalk version 3.0 (http://mirwalk.umm.uni-heidelberg.de) were used to identify the target gene of miR-15b-5p. The starbase 2.0 database online tool was used to analyze the association between miR-15b-5p and the predicted targets. Gene Expression Profiling Interactive Analysis 2 (GEPIA2; http://gepia.cancer-pku.cn/) was used to assess the expression of the target gene in THCA. Subsequently, log-rank test was performed to analyze the differences of the overall survival time between patients with high and low expression of the target gene.

Cell lines and culture. Human THCA cell lines FTC133 (derived from follicular THCA; cat. no. ECACC 94060901), SW1736 (derived from anaplastic THCA; cat. no. CLS 300453) and K1 (derived from a primary papillary THCA; cat. no. ECACC 92030501; authenticated by Shanghai Genechem Co., Ltd. using short tandem repeat profile analysis), as well as the thyroid epithelial cell line Nthy-ori3-1 (Nthy3; cat. no. SA 90011609) were purchased from the European Collection of Cell Cultures, CLS Cell Lines Service GmbH or Sigma-Aldrich (Merck KGaA). FTC133 and K1 were maintained in DMEM/modified HAM-F12 medium (Invitrogen; Thermo Fisher Scientific, Inc.), while SW1736 and Nthy3 were cultured in RPMI-1640 medium (Thermo Fisher Scientific,
Inc.) at $37^{\circ} \mathrm{C}$ with an atmosphere of $5 \% \mathrm{CO}_{2}$. Both of mediums were supplemented with 10\% FBS (Gibco; Thermo Fisher Scientific, Inc.).

Cell transfection. miR-15b-5p agomir (5'-UAGCAGCAC AUCAUGGUUUACA-3', $20 \mathrm{nM}$ ), miR-15b-5p antagomir (5'-TGTAAAUUATGATGTGUTGUTA-3', $50 \mathrm{nM}$ ) and corresponding negative controls $(\mathrm{NC}, 50 \mathrm{nM})$, pcDNA3.1 and pcDNA3.1-GDI2 (1 $\mu \mathrm{g} / \mathrm{well})$, and small interfering RNA (si)-NC and si-GDI2 (5'-CCAAGTTCCTTATGGCTAA-3', $50 \mathrm{nM}$ ) were designed and synthesized by Guangzhou RiboBio Co., Ltd. Then, the transfection assays were performed using Lipofectamine $^{\circledR} 3000$ (Invitrogen; Thermo Fisher Scientific, Inc.) following the manufacturer's instructions. The cells were harvested for subsequent experiments, $24 \mathrm{~h}$ post transfection.

Assessment of cell viability using Cell Counting Kit (CCK-8) assay. SW1736 and K1 cells with different treatments were plated into 24 -well plates $(3,000$ cells/well) in DMEM containing $10 \% \mathrm{FBS}$ at $37^{\circ} \mathrm{C}$. Then, $10 \mu \mathrm{l}$ CCK- 8 regent (Beijing Solarbio Science \& Technology Co., Ltd.) was added into each well and mixed for $1.5 \mathrm{~h}$ at $37^{\circ} \mathrm{C}$, and the cell viability was measured according to the manufacture's protocol every $24 \mathrm{~h}$, from 0 to $96 \mathrm{~h}$. A microplate reader was utilized to measure the optical density value at $450 \mathrm{~nm}$.

Transwell assays. Transwell assays were performed to examine cell invasion. In brief, $100 \mu \mathrm{l}$ Matrigel, which was incubated in serum-free medium overnight at $37^{\circ} \mathrm{C}$, was added to the upper Transwell chamber (EMD Millipore) and subsequently inserted into 24 -well plates. The cells were cultured at $37^{\circ} \mathrm{C}$ for 4-6 h. Then, $500 \mu \mathrm{l}$ serum-free medium was added into lower chamber and incubated for $1.5 \mathrm{~h}$ at $37^{\circ} \mathrm{C}$. Subsequently, $100 \mu \mathrm{l}$ cell suspension ( $1 \times 10^{5}$ transfected cells) was prepared using medium without serum and plated into the upper chamber, with $500 \mu \mathrm{l}$ complete culture solution plated into the lower chamber. After $24 \mathrm{~h}$, the chambers were removed and washed with PBS, followed by fixation with $4 \%$ paraformaldehyde for $30 \mathrm{~min}$ at room temperature. Staining was performed using $0.1 \%$ crystal violet for $20 \mathrm{~min}$ at room temperature and the number of invading cells was counted under a light microscope at x200 magnification.

Reverse transcription-quantitative PCR (RT-qPCR). Total RNA of the cells was extracted using TRIzol ${ }^{\circledR}$ (Invitrogen; Thermo Fisher Scientific, Inc.). RT was conducted using different kits: For mRNAs, PrimeScript RT Reagent kit (Takara Bio, Inc.); and for miRNAs, MiScript RT kit (Qiagen, Inc.). The RT reaction conditions were as follows: Incubation at $42^{\circ} \mathrm{C}$ for $50 \mathrm{~min}$, followed by incubation at $85^{\circ} \mathrm{C}$ for $5 \mathrm{~min}$ to terminate the reaction. Subsequently, RT-qPCR was performed with SYBR Premix Ex Taq II (Takara Bio, Inc.) for mRNA and miScript SYBR-Green PCR kit (Qiagen, Inc.) for miRNA in a 7900HT RT PCR system (Applied Biosystems; Thermo Fisher Scientific, Inc.). GAPDH and U6 were used the internal references of mRNA and miRNA, respectively. The following thermocycling conditions were used for qPCR: Initial denaturation at $95^{\circ} \mathrm{C}$ for $30 \mathrm{sec} ; 40$ cycles of $95^{\circ} \mathrm{C}$ for $5 \mathrm{sec}, 60^{\circ} \mathrm{C}$ for $30 \mathrm{sec}$. The relative mRNA expression was calculated with 
A

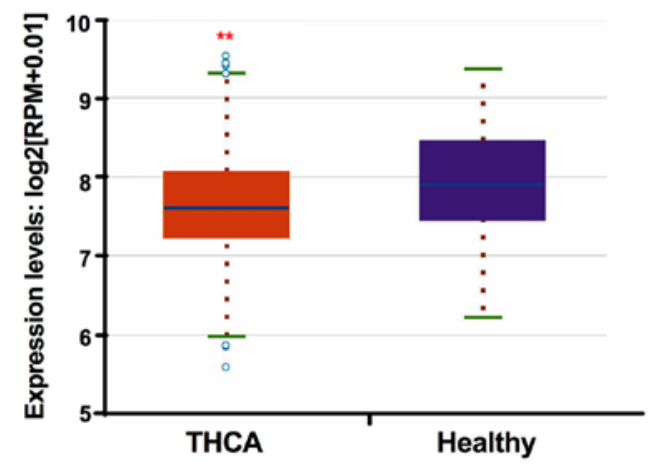

B

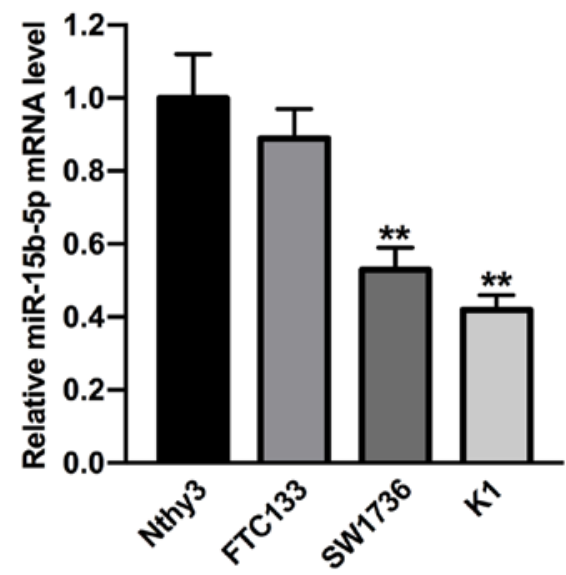

C

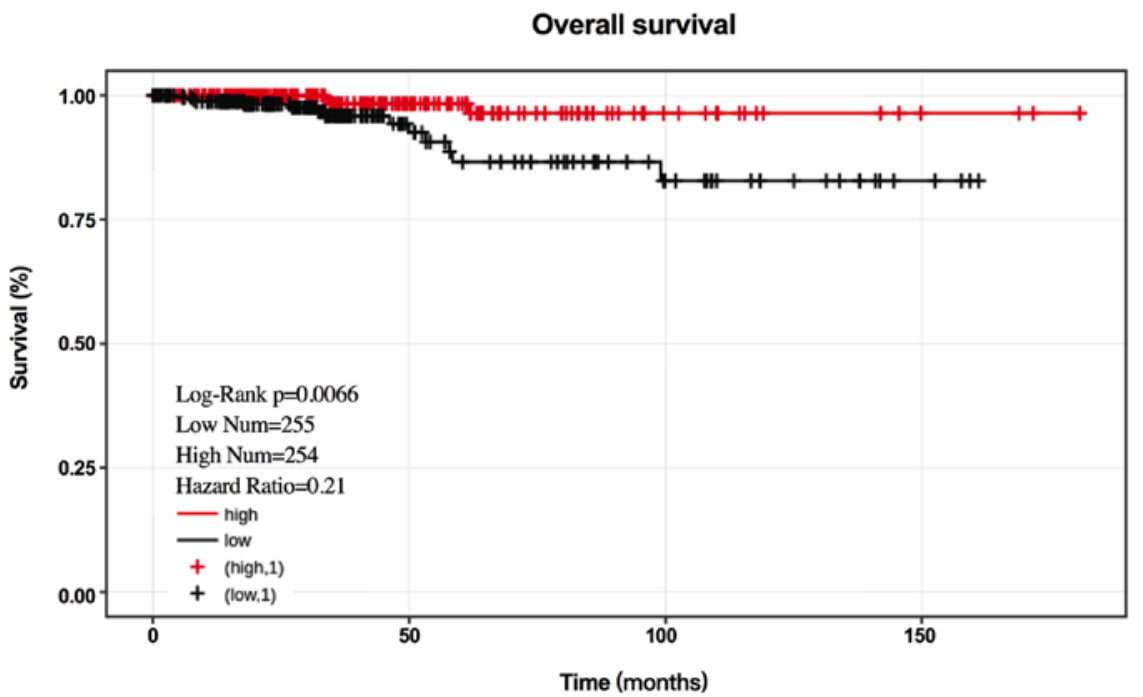

Figure 1. Expression of miR-15b-5p and its association with overall survival of patients with THCA. (A) Expression of miR-15-5b-5p was lower in patients with THCA $(n=509)$ compared with healthy controls $(n=58)$ according to starbase 2.0 database. ${ }^{* *} \mathrm{P}<0.01$ vs. healthy controls. (B) Reverse transcription-quantitative PCR analysis identified the downregulation of miR-15b-5p in THCA cells compared with normal thyroid epithelial cells. All experiments were repeated three times. ${ }^{* *} \mathrm{P}<0.01$, vs. Nthy3 cells. (C) Patients with low expression of miR-15b-5p had a poorer prognosis compared with patients with high expression of miR-15b-5p (log rank, $\mathrm{P}<0.01)$. miR, microRNA; THCA, thyroid carcinoma.

$2^{-\Delta \Delta \mathrm{Cq}}$ analysis method (32). Primers used were as follows: GDI2 forward (F), 5'-TTTCGTAAGCTTATGGACGAGGAA TACGATGT-3' and reverse (R), 5'-TTTCGTCTCGAGGCT GTTAGTCTTCCCCATAG-3'; GAPDH F, 5'-CAACTTTGG CATCGTGGAAGGG-3' and R, 5'-CAACGGATACATTGG GGGTAGG-3'. miR-15b-5p F, 5'-TAGCAGCACATCATG GTTTACA-3' and R, 5'-TGCGTGTCGTGGAGTC-3'; and U6 F, 5'-CTCGCTTCGGCAGCACA-3' and R, 5'-AACGCTTCA CGAATTTGCGT-3'.

Western blot analysis. Total protein was isolated using RIPA buffer (Beijing Solarbio Science \& Technology Co., Ltd., with protease inhibitor) and the protein concentration was measured with a bicinchoninic acid protein assay kit (Sigma-Aldrich; Merck KGaA). Then, $20 \mu \mathrm{g}$ protein/lane was added into each well of the vertical electrophoresis tank and separated using 10\% SDS-PAGE. Subsequently, the protein was transferred onto the PVDF membrane. After being blocked with $5 \%$ skim-milk for $1 \mathrm{~h}$ at room temperature, the membranes were incubated with the primary antibodies as follows: Matrix metalloproteinase (MMP)2 (1:300; cat. no. sc-13594; Santa Cruz Biotechnology, Inc.), MMP9 (1:300; cat. no. sc-21733; Santa Cruz Biotechnology, Inc.), GDI2 (1:1,000; cat. no. ab234986; Abcam) and GAPDH (1:4,000; cat. no. sc-47724; Santa Cruz Biotechnology) at $4^{\circ} \mathrm{C}$ for overnight. The membranes were washed three times with $0.1 \%$ TBS-Tween-20 (each time $5 \mathrm{~min}$ ) and mixed with goat anti-rabbit/mouse horseradish peroxide-conjugated secondary antibodies (1:5,000; cat. nos. ab205718 and ab205719; Abcam) at room temperature for $1 \mathrm{~h}$. Then, the membranes were rinsed with TBS and developed using ECL (Thermo Fisher Scientific, Inc.). Protein expression was semi-quantified using Quantity One software (version 4.6.6, Bio-rad Laboratories, Inc.) and normalized to GAPDH.

Dual-luciferase assay. The 3'-UTR of GDI2, harboring either the wild-type (WT) or mutant (MT) miR-15b-5p binding site was cloned into the downstream of pmirGLO Dual-Luciferase 
A

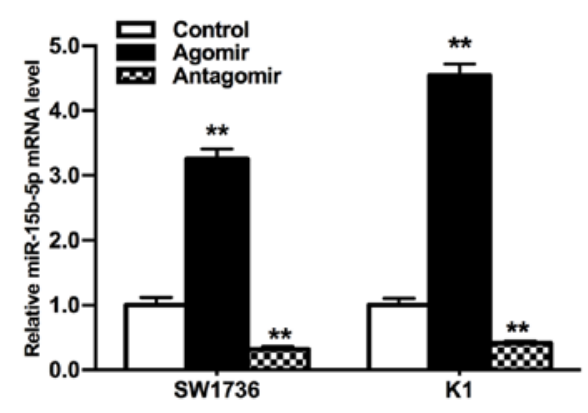

C

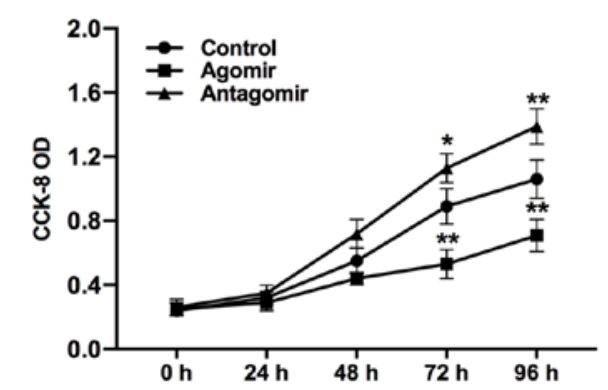

E

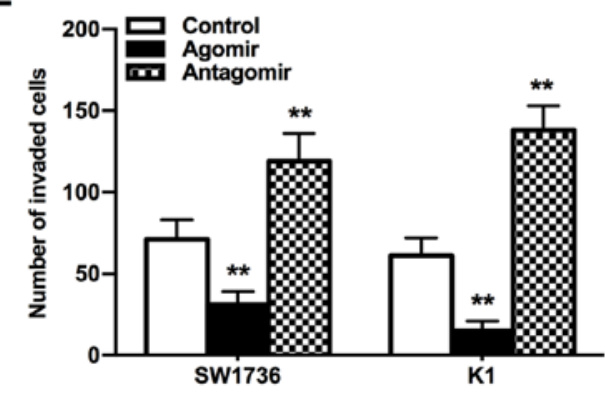

B

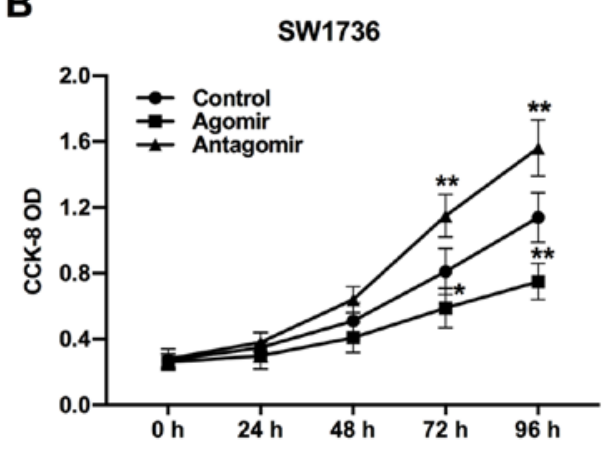

D

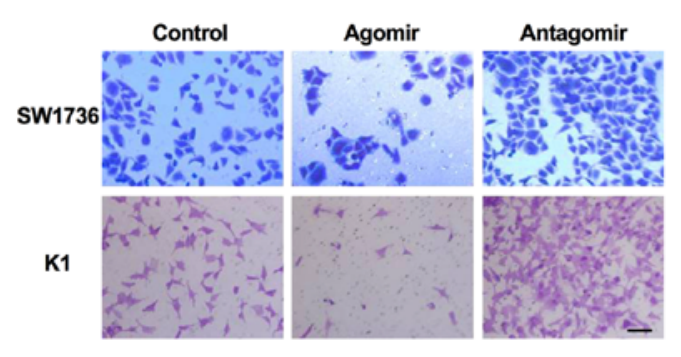

Figure 2. Influences of miR-15b-5p on the proliferation and invasion of thyroid carcinoma cells. (A) miR-15b-5p expression was overexpressed or knocked down using miR-15b-5p agomir or antagomir in SW1736 and K1 cells, respectively. miR-15b-5p agomir or antagomir inhibited or promoted the proliferation of (B) SW1736 and (C) K1 cells, respectively. (D) Cell invasion of SW1736 and K1 cells was (E) suppressed or increased by miR-15b-5p agomir or antagomir, respectively. Scale bar, $200 \mu \mathrm{m}$. All experiments were repeated three times. Control group cells were treated with the mixture of miR-15b-5p agomir control and miR-15b-5p antagomir control. ${ }^{*} \mathrm{P}<0.05$ vs. control group; ${ }^{* *} \mathrm{P}<0.01$ vs. control group. CCK-8, Cell Counting Kit-8; OD, optical density; miR, microRNA.

vector (Promega Corporation) to generate the double luciferase reporter plasmid. Subsequently, the co-transfections of $20 \mathrm{nM}$ miR-15b-5p agomir or NC and $100 \mathrm{ng}$ double luciferase reporter plasmid into the cells were performed utilizing Lipofectamine ${ }^{\circledR}$ 2000 (Invitrogen; Thermo Fisher Scientific, Inc.). Luciferase activities were measured using the Dual-Luciferase ${ }^{\circledR}$ Reporter Assay System (Promega Corporation) after being transfected for $48 \mathrm{~h}$. The results were normalized to the Renilla luciferase activity and expressed as relative luciferase activity (firefly luminescence/Renilla luminescence).

Statistical analysis. SPSS 22.0 (IBM Corp.) was used to analyze the data, while GraphPad Prism 6.0 (GraphPad Software, Inc.) was used to create the figures. Each experiment was repeated $\geq 3$ times. The differences between two groups were estimated using an unpaired Student's t-test, and $\geq 3$ groups were analyzed using one-way ANOVA analysis followed by Tukey's multiple comparisons post hoc test. $\mathrm{P}<0.05$ was considered to indicate a statistically significant difference.

\section{Results}

Expression and prognostic value of miR-15b-5p in THCA. By searching the starbase2.0 database, it was found that miR-15b-5p expression was reduced in THCA compared with healthy individuals (Fig. $1 \mathrm{~A} ; \mathrm{P}<0.01$ ). To assess this result, miR-15b-5p expression was determined in human the THCA cell lines FTC133, SW1736 and K1, and a normal cell line Nthy3. The results demonstrated that miR-15b-5p expression was significantly decreased expressed in SW1736 and K1 cells compared with Nthy 3 cells (Fig. $1 \mathrm{~B} ; \mathrm{P}<0.01$ ). While the expression of miR-15b-5p was lower in FTC133 cells compared with Nthy 3 cells, the difference was not significant $(P>0.05)$. Therefore, SW1736 and K1 cells were used in the subsequent experiments.

To analyze the impacts of miR-15b-5p on the overall survival of patients with THCA, Kaplan-Meier analysis was performed. In total, two groups (high miR-15b-5p expression, $n=254$; low miR-15b-5p expression, $n=255$ ) were 
determined based on the median expression of miR-15b-5p in THCA tissues. It was demonstrated that patients with high miR-15b-5p expression had a longer overall survival time compared with patients with low miR-15b-5p expression (Fig. $1 C ; \mathrm{P}<0.01$ ). Thus, the results suggested that miR-15b-5p was downregulated in THCA, and lower miR-15b-5p expression was related to the tumor progression and poor prognosis of THCA.

Suppression of THCA cell proliferation and invasion induced by $m i R-15 b-5 p$. To estimate the efficacy of miR-15b-5p in THCA progression, the expression of miR-15b-5p in SW1736 and $\mathrm{K} 1$ cells was altered using miR-15b-5p agomir or antagomir. Then, the proliferative and invasive abilities of THCA cells were evaluated using CCK-8 and Transwell assays, respectively. miR-15b-5p expression was significantly increased in miR-15b-5p agomir group, while it was significantly reduced in miR-15b-5p antagomir group compared with the control group (Fig. 2A; $\mathrm{P}<0.01$ ). CCK-8 assay results indicated that the THCA cell proliferation was significantly accelerated by the miR-15b-5p antagomir, but was suppressed by the miR-15b-5p agomir compared with the control (Fig. $2 \mathrm{~B}$ and $\mathrm{C} ; \mathrm{P}<0.05$ and $\mathrm{P}<0.01)$. According to Transwell assay results, the miR-15b-5p agomir reduced the invasion of THCA cells, while the invasive ability of those treated by miR-15b-5p antagomir was increased (Fig. 2D and E; $\mathrm{P}<0.01$ ). Therefore, it was indicated that miR-15b-5p may be an inhibitor in the tumor development of THCA.

miR-15b-5p regulates the expression levels of invasionassociated proteins MMP2 and MMP9. To investigate how miR-15b-5p exerted its effects on cell invasion, the expression levels of invasion-associated proteins MMP2 and MMP9 were measured using western blot analysis. Overexpression of miR-15b-5p significantly reduced the protein expression levels of MMP2 and MMP9 compared with the control (Fig. 3; P<0.01). Thus, miR-15b-5p may suppress the invasion of THCA cells by inhibiting the expression levels of MMP2 and MMP9.

GDI2 is targeted by miR-15b-5p and related to the prognosis of patients with THCA. Next, the target genes of miR-15b-5p were examined using TargetScan, miRanda and miRWalk online tools, and GDI2 was obtained via the intersection of these databases. Then, the relevance of miR-15b-5p and GDI2 expression levels were analyzed using starbase website. The results demonstrated that miR-15b-5p expression was negatively associated with GDI2 expression (Fig. 4A; $\mathrm{P}<0.05$ ). Moreover, there was a slight increase in GDI2 expression in patients with THCA compared with healthy controls, as determined via the online analysis tool of the GEPIA website (Fig. 4B; P>0.05), but the high expression of GDI2 was found to predict a poor prognosis of patients with THCA (Fig. 4C; $\mathrm{P}<0.01$ ), suggesting the involvement of GDI2 in THCA progression.

Subsequently, the effect of miR-15b-5p on the expression of GDI2 in THCA cells were measured using RT-qPCR and western blot analyses. The miR-15b-5p agomir repressed the relative mRNA and protein expression of GDI2, while the miR-15b-5p antagomir increased this expression
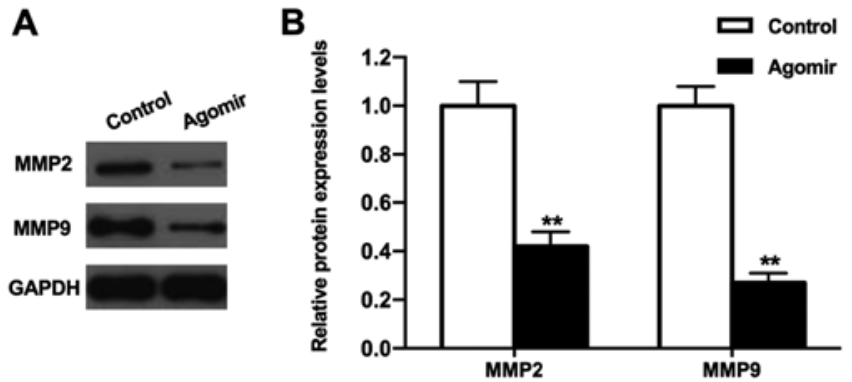

Figure 3. Expression levels of invasion-associated proteins MMP2 and MMP9 in THCA cells. (A) Western blot results indicated that (B) miR-15b-5p agomir could significantly reduce the expression levels of MMP2 and MMP9 in SW1736 cells. All experiments were repeated three times. Control group cells were treated with the miR-15b-5p agomir control. ${ }^{* *} \mathrm{P}<0.01$ vs. control. miR, microRNA; MMP, matrix metalloproteinase.

(Fig. 4D and E; all $\mathrm{P}<0.01$ ), which indicated that the expression of GDI 2 was negatively modulated by miR-15b-5p.

To assess whether miR-15b-5p regulated GDI 2 by binding to the 3'-UTR of GDI2, a dual-luciferase reporter assay was performed. The binding sites between miR-15b-5p and GDI2 were predicted via the bioinformatics analysis as aforementioned (Fig. 4F). The luciferase activity of pmirGLO-GDI2-WT in the miR-15b-5p agomir group was significantly lower compared with the control group (Fig. 4G; P<0.01); however, the luciferase activity of pmirGLO-GDI2-MT had no significant differences between the two groups, suggesting that GDI2 may be the downstream target genes of miR-15b-5p in the tumor progression of THCA. Collectively, these results indicated that GDI2 was upregulated in THCA and may be involved in the progression of THCA modulated by miR-15b-5p.

GDI2 abolishes the effects of miR-15b-5p on the phenotype of THCA cells. To examine whether GDI2 was involved in the effects of miR-15b-5p during the tumor progression, a transfection assay was conducted on the SW1736 and K1 cells with si-con and si-GDI2, as well as pcDNA3.1 and pcDNA3.1-GDI2. RT-qPCR and western blot analyses demonstrated that the mRNA and protein expression levels of GDI2 were both downregulated after the cells were transfected with si-GDI2, while these were upregulated in cells transfected with pcDNA3.1-GDI2 compared with the control (Fig. 5A-C; all $\mathrm{P}<0.01)$.

CCK-8 and Transwell assays were performed to evaluate the functions of the miR-15b-5p/GDI2 axis in the proliferation and invasion of THCA cells. It was found that the proliferation of THCA cells was increased in the miR-15b-5p agomir + pcDNA3.1-GDI2 group compared with the miR-15b-5p agomir group, but the proliferative ability in miR-15b-5p antagomir + si-GDI2 group was significantly reduced compared with the miR-15b-5p antagomir group (Fig. 5D and E; all $\mathrm{P}<0.01$ ). Furthermore, the invasive abilities of THCA cells in the miR-15b-5p agomir + pcDNA3.1-GDI2 group were significantly enhanced compared with the miR-15b-5p agomir group, while the knockdown of GDI2 and miR-15b-5p together suppressed the invasion of THCA cells compared with the miR-15b-5p antagomir group (Fig. 5F and G; all $\mathrm{P}<0.01$ ). It was also found that the proliferative and invasive abilities of THCA cells in miR-15b-5p agomir + pcDNA3.1-GDI2 group 
A

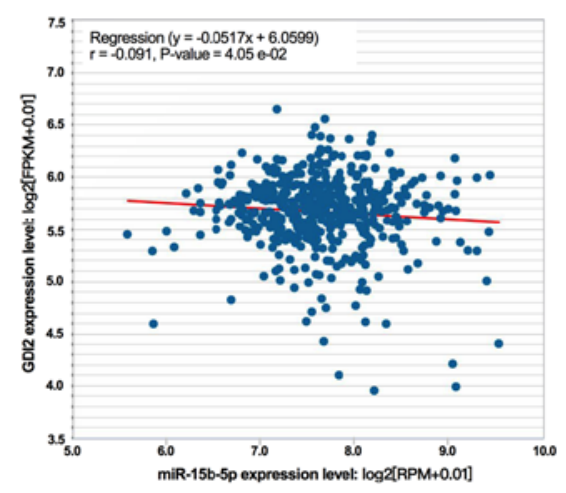

B

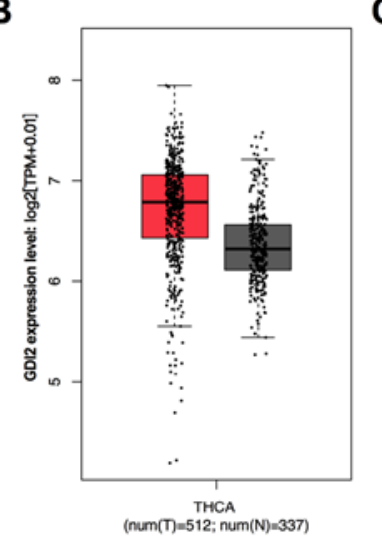

C

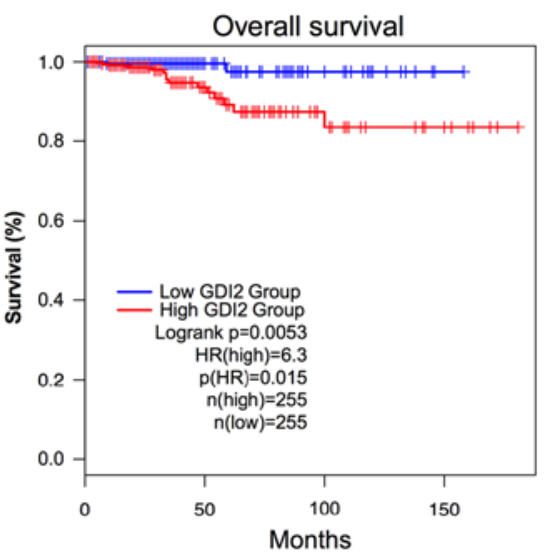

D

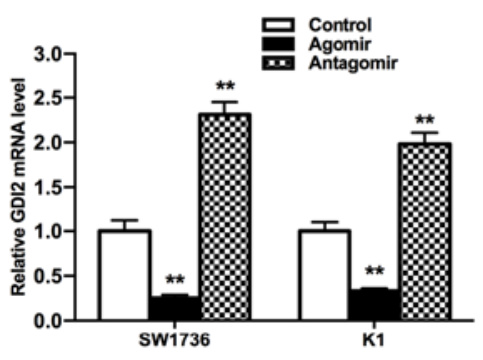

$\mathbf{E}$

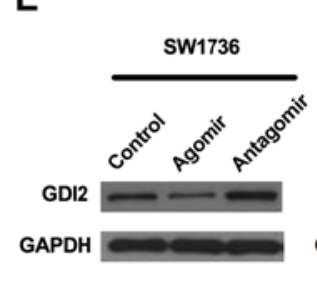

GDI2 GAPDH
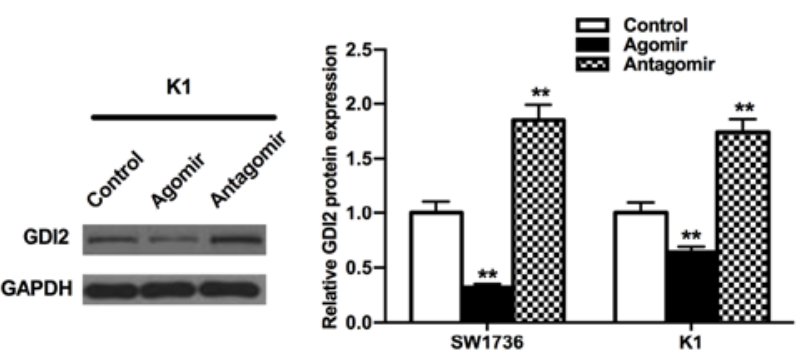

$\mathbf{F}$

$$
\begin{array}{rc}
\text { GDI2: } & \text { 5' guguagCUAUG-UGCUGCUGCUg 3' } \\
|:|:|||||||||||| \\
\text { miR-15b-5p: } & \text { 3' acauUUGGUACUAC-ACGACGAu 5' } \\
\text { MT GDI2: } & \text { 5' guguaGCUUAC-UUCACGACCUg 3' }
\end{array}
$$

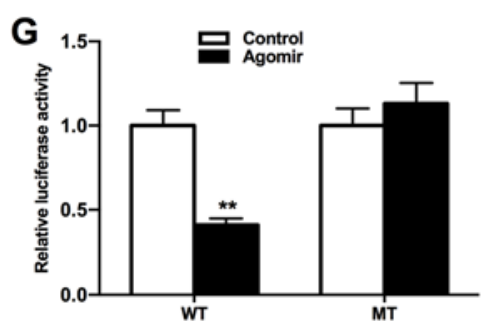

Figure 4. Expression of GDI2 and its association with miR-15b-5p. (A) miR-15b-5p expression was negatively associated with GDI2 expression. Data analysis from starbase 2.0 database. (B) GDI2 expression in thyroid tissues from patients with THCA and healthy controls. Data analysis from Gene Expression Profiling Interactive Analysis. Red box presented patients with THCA (T), and grey box presented healthy (N) controls. (C) High expression of GDI2 could lead to a shorter overall survival time for patients with THCA. (D) mRNA and (E) protein expression levels of GDI2 were repressed or enhanced by miR-15b-5p agomir or antagomir in SW1736 and K1 cells, respectively. Control group cells were treated with the mixture of miR-15b-5p agomir control and miR-15b-5p antagomir control. (F) Binding sites between GDI2 and miR-15b-5p. (G) miR-15b-5p agomir decreased the luciferase activity of pmirGLO-GDI2-WT but had no influence on the luciferase activity of pmirGLO-GDI2-MT. Control group cells were treated with the miR-15b-5p agomir control. All experiments were repeated three times. ${ }^{* *} \mathrm{P}<0.01$ vs. control group. WT, wild-type; MT, mutant; miR, microRNA; GDI2, GDP dissociation inhibitor 2; THCA, thyroid carcinoma; HR, hazard ratio.

and miR-15b-5p antagomir + si-GDI2 group were both almost return to the level of control group.

To determine the relative expression levels of the invasion-associated proteins MMP2 and MMP9, western blot analysis was conducted. MMP2 and MMP9 expression levels were both increased in the miR-15b-5p agomir + pcDNA3.1-GDI2 group compared with the miR-15b-5p agomir group in SW1736 cells (Fig. $5 \mathrm{H} ; \mathrm{P}<0.01$ ). In addition, the expression levels of MMP2 and MMP9 were both decreased in miR-15b-5p antagomir + si-GDI2 group compared with the miR-15b-5p antagomir group in $\mathrm{K} 1$ cells (Fig. 5I; $\mathrm{P}<0.01$ ). It was also demonstrated that the expression levels of MMP2 and MMP9 could be both recovered to similar levels as the control in miR-15b-5p agomir + pcDNA3.1-GDI2 group or miR-15b-5p antagomir + si-GDI2 group ( $>00.05)$. Collectively, the results suggested that miR-15b-5p could inhibit cell proliferation and invasion, and regulate the expression levels of MMP2 and MMP9 via targeting GDI2.

\section{Discussion}

Recently, the incidence of THCA has been continually increasing and is higher compared with other cancer types (33). Despite THCA having a relative low mortality rate, patients with advanced THCA still have an undesirable prognosis (34-36). Thus, identifying novel molecular markers of THCA is of importance for its effective treatment. The present study identified the low expression of miR-15b-5p in THCA, which was associated with a shorter overall survival time of patients with THCA, as well as promoted the proliferation and invasion of THCA cells via regulating the expression levels of the invasion-associated proteins MMP2 and MMP9.

Previous studies have reported that miRNAs serve vital roles in the oncogenesis of THCA due to their effects on the regulation of biological features in THCA (37). For example, miR-136-5p, miR-26a, miR-214 and miR-1266 are abnormally expressed in THCA and modulate the tumor progression of THCA (37-40). miR-15b-5p is the dominant 
A

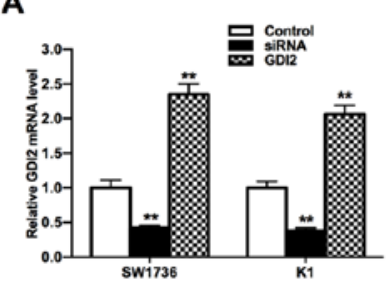

D

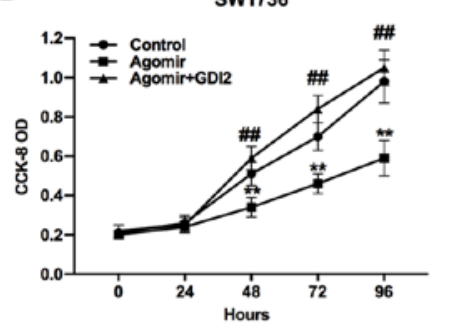

B

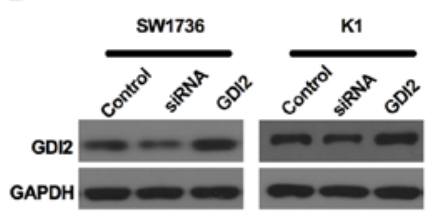

C

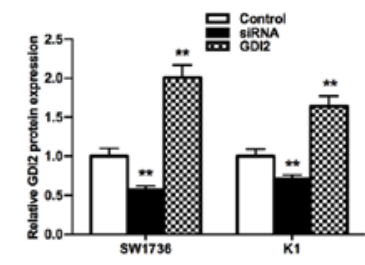

K1

E

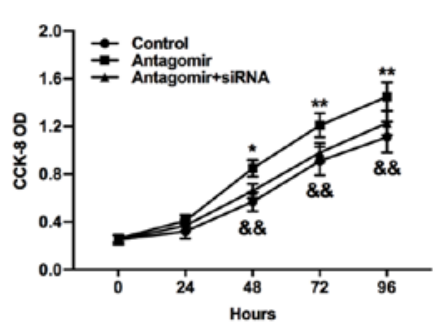

$\mathbf{F}$

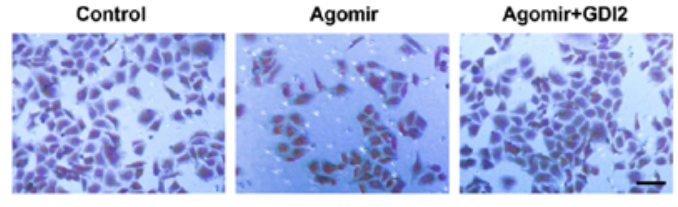

sw1736
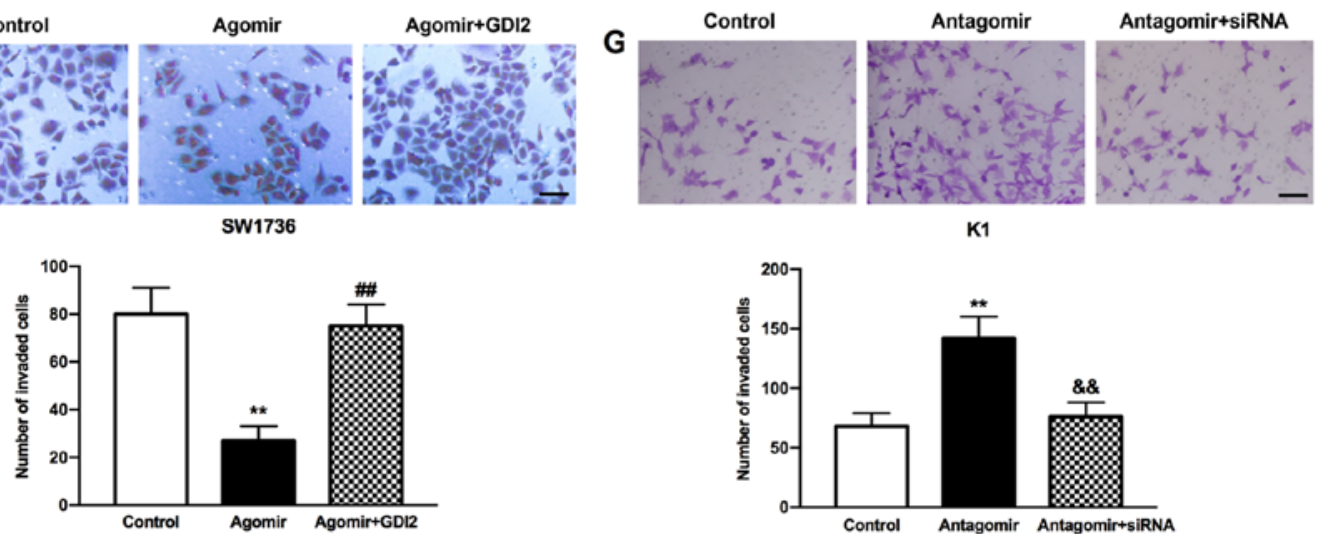

K1

H

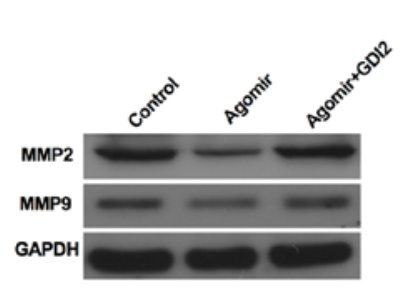

I
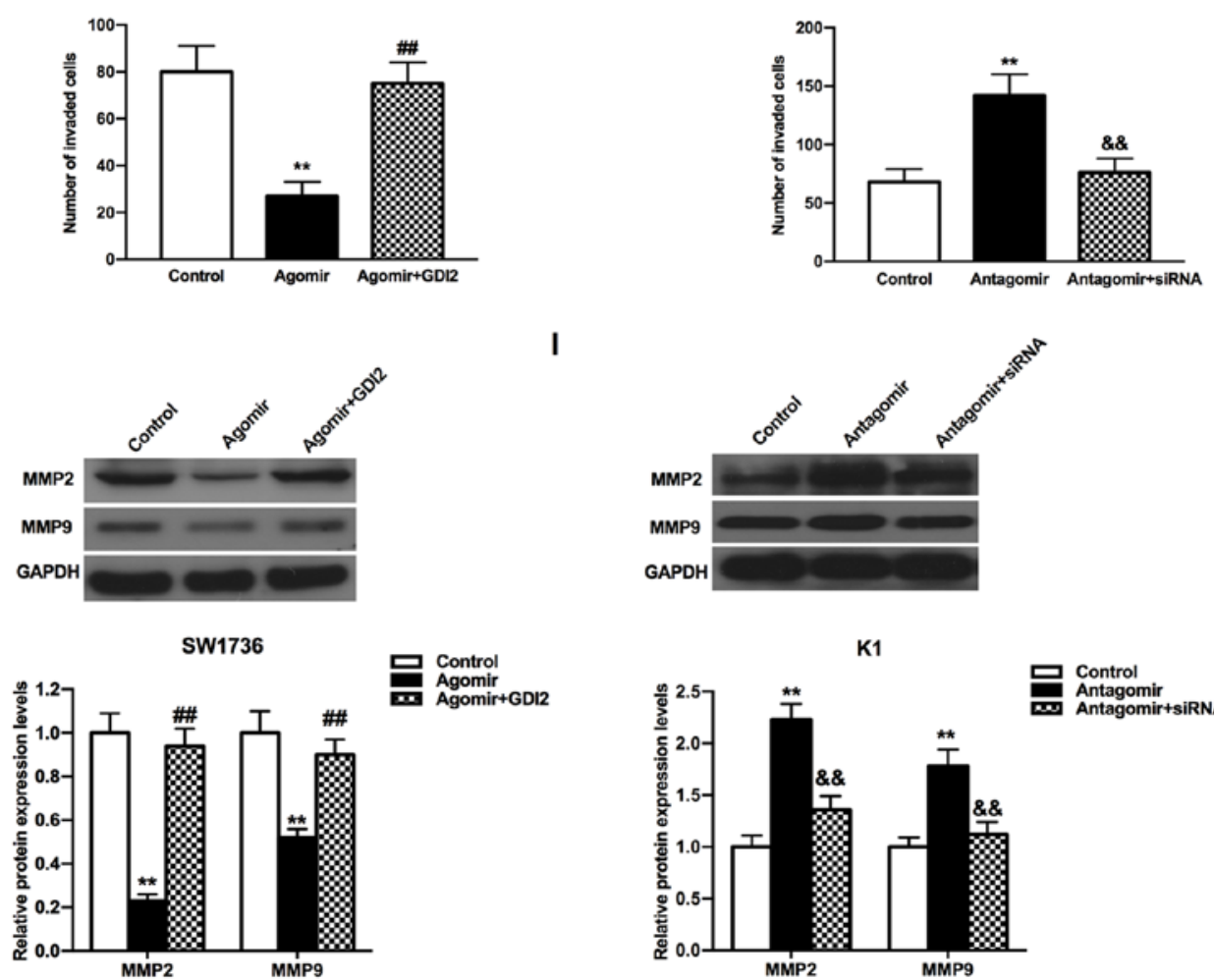

Figure 5. Impacts of miR-15b-5p and GDI2 on the proliferation and invasion of THCA cells. GDI2 expression was reduced or induced by si-GDI2 or pcDNA3.1-GDI2 in SW1736 and K1 cells both at (A) mRNA and (B) protein levels, (C) which were semi-quantified from western blot analysis. Control group cells were treated with a mixture of the scrambled siRNAs and pcDNA3.1. Proliferation of (D) SW1736 and (E) K1 cells were attenuated or accelerated by miR-15b-5p agomir or miR-15b-5p antagomir, which was restored by pcDNA3.1-GDI2 or si-GDI2. Inhibitory or stimulative effect of miR-15b-5p agomir or miR-15b-5p antagomir on (F) SW1736 and (G) K1 cell invasion was weakened by pcDNA3.1-GDI2 or si-GDI2. Scale bar, 200 $\mu \mathrm{m}$. pcDNA3.1-GDI2 or si-GDI2 increased or decreased the expression levels of MMP2 and MMP9, which were caused by miR-15b-5p agomir or miR-15b-5p antagomir, in (H) SW1736 and (I) K1 cells. Control group cells were treated with the mixture of miR-15b-5p agomir control and pcDNA3.1 in D, F and $\mathrm{H}$, or the mixture of miR-15b-5p antagomir control and the scrambled siRNAs in $\mathrm{E}, \mathrm{G}$ and $\mathrm{I}$. All experiments were repeated three times. ${ }^{*} \mathrm{P}<0.05,{ }^{* *} \mathrm{P}<0.01 \mathrm{vs}$. control group; ${ }^{\# \prime} \mathrm{P}<0.01 \mathrm{vs}$. agomir group; ${ }^{\&} \mathrm{P}<0.01$ vs. the antagomir group. miR, microRNA; GDI2, GDP dissociation inhibitor 2; siRNA, small interfering RNA; MMP, matrix metalloproteinase; CCK-8, Cell Counting Kit-8; OD, optical density.

isoform of miR-15b, which is located on the long arm of chromosome 3 (41), and has been revealed to be dysregulated and involved in the tumor development processes of various cancer types. For instance, miR-15b-5p is upregulated in gastric cancer and facilitates tumor metastasis by targeting progestin and adipoQ receptor family member 3 (15). 
Moreover, the overexpression of miR-15b-5p has been shown to promote the proliferation and invasion of liver cancer cells via targeting axis formation inhibitor 2 (17). The upregulation of miR-15b-5p has also been reported to be involved in hepatocarcinogenesis via various mechanisms, including inducing endoplasmic reticulum stress and apoptosis by regulating Rab1A (21), and acts as a diagnostic marker in hepatocellular carcinoma (19-22). Furthermore, knockdown of miR-15b-5p could inhibit the tumor growth of prostate cancer by targeting reversion inducing cysteine rich protein with kazal motifs (23).

The inhibitory effects of miR-15b-5p have been revealed in neuroblastoma, and it can restrain tumor progression by directly targeting MYCN proto-oncogene, bHLH transcription factor (18). Luo et al (42) demonstrated that miR-15b-5p reduced proliferation, but induced apoptosis and cytotoxic activities of PC12 cells. Moreover, Ahmad et al (43) reported that miR-15b-5p could be a potential biomarker for the treatment of squamous cell carcinoma. miR-15p-5b expression also exhibits a downward trend in osteosarcoma (44), colon cancer (45) and squamous cell carcinoma (46). Consistent with the decrease in miR-15b-5p expression in these aforementioned cancer types, the present results suggested that miR-15b-5p was downregulated in THCA. Furthermore, miR-15b-5p was found to inhibit cell proliferation and invasion, which was consistent with the suppressive effect observed in neuroblastoma (18). MMP2 and MMP9 are important inflammatory biomarkers and the increased expression of these factors has been shown to facilitate cell invasion in THCA $(47,48)$. In line with this finding, the current results indicated that miR-15b-5p suppressed THCA cell invasion via regulating the expression levels of MMP2 and MMP9. To the best of our knowledge, the present study was the first to investigate the functions of miR-15b-5p in THCA and may provide a novel therapy target site for THCA.

miRNAs can mediate the expression of the target genes via binding to their 3'-UTR (49). Previous studies have revealed that miR-15b-5p can exert effects in the tumor progression via targeting different genes, such as Rab1A, MYCN and Axin2 (17,18,21). Therefore, the present study examined the potential targets of miR-15b-5p and GDI2 was recognized as the target gene of miR-15b-5p. GDI2 has been reported to serve a key role in several cancer types. For instance, the upregulation of GDI2 has been identified in pancreatic cancer (26) and esophageal squamous cell carcinoma (30), indicating that GDI2 could be potential molecular targets or biomarkers for these cancer types. In addition, the downregulation of GDI2 has been observed in ovarian cancer and it may be a potential biomarker of the paclitaxel-resistant ovarian cancer for tailored cancer therapy (29). The present results identified a high expression of GDI 2 in THCA and its negative association with the overall survival of patients with THCA. It was also found that the inhibitory effects of miR-15b-5p on the proliferative and invasive abilities of THCA cells were both weakened by the overexpression of GDI2. Furthermore, GDI2 increased the low expression levels of MMP2 and MMP9, which were inhibited by the overexpression of miR-15b-5p. Therefore, it was speculated that the regulatory roles of miR-15b-5p were mediated by GDI 2 in THCA. However, the mechanism via which the miR-15b-5p/GDI2 axis communicates with
MMP2 and MMP9 requires further investigation. While the expression levels of miRNAs-15b-5 and GDI2 in the THCA were changed significantly compared with healthy tissues, the extensive overlapping between the individual values suggested the use of miRNAs-15b-5 and GDI2 values as tumor biomarkers should be moderated.

In conclusion, the current results demonstrated that miR-15b-5p could prevent the progression of THCA by binding and negatively regulating GDI2 expression. Therefore, these preliminary findings indicated the functions and underlying mechanisms of miR-15b-5p in THCA mainly via in vitro cells experiments, and provided a novel potential therapeutic target for THCA treatment in the future. However, additional studies are required to verify the validity of aforementioned conclusions in vivo.

\section{Acknowledgements}

Not applicable.

\section{Funding}

The present study was funded by the Research Award Fund for Outstanding Young and Middle-Aged Scientists in Shandong Province (grant no. BS2010YY059).

\section{Availability of data and materials}

The datasets used and/or analyzed during the present study are available from the corresponding author on reasonable request.

\section{Authors' contributions}

JZ designed the study and wrote the manuscript. JQ, HF, FY, WZ and LX performed the experiments and statistical analysis. All authors read and approved the final manuscript.

\section{Ethics approval and consent to participate}

Not applicable.

\section{Patient consent for publication}

Not applicable.

\section{Competing interests}

The authors declare that they have no competing interests.

\section{References}

1. Ward EM, Sherman RL, Henley SJ, Jemal A, Siegal DA, Feuer EJ, Firth AU, Kohler BA, Scott S et al.: Annual report to the nation on the status of cancer, featuring cancer in men and women age 20-49 years. J Natl Cancer Inst 111: 1279-1297, 2019

2. Torre LA, Bray F, Siegel RL, Ferlay J, Lortet-Tieulent J and Jemal A: Global cancer statistics, 2012. CA Cancer J Clin 65: 87-108, 2015.

3. Huk DJ, Ashtekar A, Magner A, La Perle K and Kirschner LS: Deletion of Rap1b, but not Rap1a or Epac1, reduces protein kinase A-mediated thyroid cancer. Thyroid 28: 1153-1161, 2018. 
4. Bray F, Ferlay J, Soerjomataram I, Siegel RL, Torre LA and Jemal A: Global cancer statistics 2018: GLOBOCAN estimates of incidence and mortality worldwide for 36 cancers in 185 countries. CA Cancer J Clin 68: 394-424, 2018.

5. Luzon-Toro B, Fernandez RM, Villalba-Benito L, Torroglosa A, Antinolo $\mathrm{G}$ and Borrego S: Influencers on thyroid cancer onset: Molecular genetic basis. Genes (Basel) 10: 913, 2019.

6. Tella SH, Kommalapati A, Esquivel MA and Correa R: Potential role of metabolic intervention in the management of advanced differentiated thyroid cancer. Front Oncol 7: 160, 2017.

7. Ma Y and Sun Y: miR-29a-3p inhibits growth, proliferation, and invasion of papillary thyroid carcinoma by suppressing NF-kB signaling via direct targeting of OTUB2. Cancer Manag Res 11: $13-23,2018$.

8. McKenna LB, Schug J, Vourekas A, McKenna JB, Bramswig NC Friedman JR and Kaestner KH: MicroRNAs control intestinal epithelial differentiation, architecture, and barrier function. Gastroenterology 139: 1654-1664, 2010.

9. Wojtas B, Ferraz C, Stokowy T, Hauptmann S, Lange D, Dralle H, Musholt T, Jarzab B, Paschke R and Eszlinger M: Differential miRNA expression defines migration and reduced apoptosis in follicular thyroid carcinomas. Mol Cell Endocrinol 388: 1-9, 2014.

10. Guo H, Ingolia NT, Weissman JS and Bartel DP: Mammalian microRNAs predominantly act to decrease target mRNA levels. Nature 466: 835-840, 2010

11. Ling H, Fabbri $M$ and Calin GA: MicroRNAs and other non-coding RNAs as targets for anticancer drug development. Nat Rev Drug Discov 12: 847-865, 2013.

12. Takahashi RU, Prieto-Vila M, Hironaka A and Ochiya T: The role of extracellular vesicle microRNAs in cancer biology. Clin Chem Lab Med 55: 648-656, 2017.

13. Gao XB, Chen CL, Tian ZL, Yuan FK and Jia GL: MicroRNA-791 is an independent prognostic factor of papillary thyroid carcinoma and inhibits the proliferation of PTC cells. Eur Rev Med Pharmacol Sci 22: 5562-5568, 2018.

14. Mishra S, Yadav T and Rani V: Exploring miRNA based approaches in cancer diagnostics and therapeutics. Crit Rev Oncol Hematol 98: 12-23, 2016.

15. Zhao C,Li Y, Chen G, Wang F, Shen Z and Zhou R: Overexpression of miR-15b-5p promotes gastric cancer metastasis by regulating PAQR3. Oncol Rep 38: 352-358, 2017.

16. Rissland OS, Hong SJ and Bartel DP: MicroRNA destabilization enables dynamic regulation of the miR-16 family in response to cell-cycle changes. Mol Cell 43: 993-1004, 2011.

17. Dong Y, Zhang N, Zhao S, Chen X, Li F and Tao X: miR-221-3p and miR-15b-5p promote cell proliferation and invasion by targeting Axin2 in liver cancer. Oncol Lett 18: 6491-6500, 2019.

18. Chava S, Reynolds PC, Pathania AS, Gorantla S, Poluektova LY, Coulter DW, Gupta SC, Pandey MK and Challagundla KB: miR-15a-5p, miR-15b-5p, and miR-16-5p inhibit tumor progression by directly targeting MYCN in neuroblastoma. Mol Oncol 14: 180-196, 2020

19. Pan WY, Zeng JH, Wen DY, Wang JY, Wang PP, Chen G and Feng ZB: Oncogenic value of microRNA-15b-5p in hepatocellular carcinoma and a bioinformatics investigation. Oncol Lett 17: 1695-1713, 2019.

20. Li H, Zhang J, Lee MJ, Yu GR, Han X and Kim DG: OIP5, a target of miR-15b-5p, regulates hepatocellular carcinoma growth and metastasis through the $\mathrm{AKT} / \mathrm{mTORC} 1$ and b-catenin signaling pathways. Oncotarget 8: 18129-18144, 2017.

21. Yang Y, Hou N, Wang X, Wang L, Chang S, He K, Zhao Z, Zhao $X$, Song $T$ and Huang C: miR-15b-5p induces endoplasmic reticulum stress and apoptosis in human hepatocellular carcinoma, both in vitro and in vivo, by suppressing Rab1 A Oncotarget 6: 16227-16238, 2015.

22. Chen Y, Chen J, Liu Y, Li S and Huang P: Plasma miR-15b-5p, miR-338-5p, and miR-764 as biomarkers for hepatocellular carcinoma. Med Sci Monit 21: 1864-1871, 2015

23. Chen R, Sheng L, Zhang HJ, Ji M and Qian WQ: miR-15b-5p facilitates the tumorigenicity by targeting RECK and predicts tumour recurrence in prostate cancer. J Cell Mol Med 22: $1855-1863,2018$

24. Theodorescu D, Sapinoso LM, Conaway MR, Oxford G, Hampton GM and Frierson HF Jr: Reduced expression of metastasis suppressor RhoGDI2 is associated with decreased survival for patients with bladder cancer. Clin Cancer Res 10: 3800-3806, 2004

25. Alcántara-Hernández R, Casas-González P and García-Sáinz JA Roles of c-Src in alpha1B-adrenoceptor phosphorylation and desensitization. Auton Autacoid Pharmacol 28: 29-39, 2008.
26. Sun ZL, Zhu Y, Wang FQ, Chen R, Peng T, Fan ZN, Xu ZK and Miao Y: Serum proteomic-based analysis of pancreatic carcinoma for the identification of potential cancer biomarkers. Biochim Biophys Acta 1774: 764-771, 2007.

27. Zhang XY, Hong SS, Zhang M, Cai QQ, Zhang MX and Xu CJ Proteomic alterations of fibroblasts induced by ovarian cancer cells reveal potential cancer targets. Neoplasma 65: 104-112, 2018.

28. Bai Z, Ye Y, Liang B, Xu F, Zhang H, Zhang Y, Peng J, Shen D, Cui Z, Zhang $Z$ and Wang S: Proteomics-based identification of a group of apoptosis-related proteins and biomarkers in gastric cancer. Int J Oncol 38: 375-383, 2011.

29. Lee DH, Chung K, Song JA, Kim TH, Kang H, Huh JH, Jung SG, Ko JJ and An HJ: Proteomic identification of paclitaxel-resistance associated hnRNP A2 and GDI 2 proteins in human ovarian cancer cells. J Proteome Res 9: 5668-5676, 2010.

30. Kashyap MK, Harsha HC, Renuse S, Pawar H, Sahasrabuddhe NA, Kim MS, Marimuthu A, Keerthikumar S, Muthusamy B, Kandasamy $\mathrm{K}$, et al: SILAC-based quantitative proteomic approach to identify potential biomarkers from the esophageal squamous cell carcinoma secretome. Cancer Biol Ther 10 796-810, 2010

31. Onda M, Emi M, Yoshida A, Miyamoto S, Akaishi J, Asaka S, Mizutani K, Shimizu K, Nagahama M, Ito K, et al: Comprehensive gene expression profiling of anaplastic thyroid cancers with cDNA microarray of 25344 genes. Endocr Relat Cancer 11: 843-854, 2004.

32. Livak KJ and Schmittgen TD: Analysis of relative gene expression data using real-time quantitative PCR and the 2(-Delta Delta $\mathrm{C}(\mathrm{T})$ ) method. Methods 25: 402-408, 2001

33. Lubitz CC, Zhan T, Gunda V, Amin S, Gigliotti BJ, Fingeret AL, Holm TM, Wachtel H, Sadow PM, Wirth LJ, et al: Circulating BRAF(V600E) Levels Correlate with Treatment in Patients with Thyroid Carcinoma. Thyroid 28: 328-339, 2018.

34. Wei WJ, Zhang GQ and Luo QY: Postsurgical management of differentiated thyroid cancer in China. Trends Endocrinol Metab 29: 71-73, 2018

35. Fu G, Polyakova O, MacMillan C, Ralhan R and Walfish PG: Programmed death-ligand 1 expression distinguishes invasive encapsulated follicular variant of papillary thyroid carcinoma from noninvasive follicular thyroid neoplasm with papillary-like nuclear features. EBioMedicine 18: 50-55, 2017.

36. Lin P, He Y, Wen DY, Li XJ, Zeng JJ, Mo WJ, Li Q, Peng JB Wu YQ, Pan DH, et al: Comprehensive analysis of the clinical significance and prospective molecular mechanisms of differentially expressed autophagy-related genes in thyroid cancer. Int J Oncol 53: 603-619, 2018.

37. Gao RZ, Que Q, Lin P, Pang YY, Wu HY, Li XJ, Chen G, He Y and Yang H: Clinical roles of miR-136-5p and its target metadherin in thyroid carcinoma. Am J Transl Res 11: 6754-6774, 2019.

38. Wu YC, Li SY and Jia YF: MicroRNA-26a suppresses the malignant biological behaviors of papillary thyroid carcinoma by targeting ROCK 1 and regulating PI3K/AKT signaling pathway. Eur Rev Med Pharmacol Sci 23: 8940-8949, 2019.

39. Liu F, Lou K, Zhao X, Zhang J, Chen W, Qian Y, Zhao Y, Zhu Y and Zhang Y: miR-214 regulates papillary thyroid carcinoma cell proliferation and metastasis by targeting PSMD10. Int J Mol Med 42: 3027-3036, 2018

40. Fu YT,Zheng HB,Zhang DQ,Zhou L and Sun H: MicroRNA-1266 suppresses papillary thyroid carcinoma cell metastasis and growth via targeting FGFR2. Eur Rev Med Pharmacol Sci 22: 3430-3438, 2018.

41. Salimi S, Noorbakhsh F, Faghihzadeh S, Ghaffarpour S and Ghazanfari T: Expression of miR-15b-5p, miR-21-5p, and SMAD7 in lung tissue of sulfur mustard-exposed individuals with long-term pulmonary complications. Iran J Allergy Asthma Immunol 18: 332-339, 2019.

42. Luo H, Li Y, Liu B, Yang Y and Xu ZD: MicroRNA-15b-5p targets ERK1 to regulate proliferation and apoptosis in rat $\mathrm{PC1} 2$ cells. Biomed Pharmacother 92: 1023-1029, 2017.

43. Ahmad P, Sana J, Slavik M, Gurin D, Radova L, Gablo NA, Kazda T, Smilek P, Horakova Z, Gal B, et al: MicroRNA-15b-5p predicts locoregional relapse in head and neck carcinoma patients treated with intensity-modulated radiotherapy. Cancer Genomics Proteomics 16: 139-146, 2019.

44. Weng Y, Shen Y, He Y, Pan X, Xu J, Jiang Y, Zhang Q, Wang S, Kong $\mathrm{F}$, Zhao S, et al: The miR-15b-5p/PDK4 axis regulates osteosarcoma proliferation through modulation of the Warburg effect. Biochem Biophys Res Commun 503: 2749-2757, 2018. 
45. Zhao C, Zhao Q, Zhang C, Wang G, Yao Y, Huang X, Zhan F, Zhu Y, Shi J, Chen J, et al: miR-15b-5p resensitizes colon cancer cells to 5 -fluorouracil by promoting apoptosis via the NF- $\mathrm{B}$ /XIAP axis. Sci Rep 7: 4194, 2017.

46. Jin X, Chen Y, Chen H, Fei S, Chen D, Cai X, Liu L, Lin B, Su H, Zhao L, et al: Evaluation of tumor-derived exosomal miRNA as potential diagnostic biomarkers for early-stage non-small cell lung cancer using next-generation sequencing. Clin Cancer Res 23: 5311-5319, 2017.

47. Gu M: IL13Ralpha2 siRNA inhibited cell proliferation, induced cell apoptosis, and suppressed cell invasion in papillary thyroid carcinoma cells. Onco Targets Ther 11: 1345-1352, 2018.
48. Huang LL, Wang Z, Cao CJ, Ke ZF, Wang F, Wang R, Luo CQ, $\mathrm{Lu} \mathrm{X}$ and Wang LT: AEG-1 associates with metastasis in papillary thyroid cancer through upregulation of MMP2/9. Int J Oncol 51: 812-822, 2017.

49. Liu ZM, Wu ZY, Li WH, Wang LQ, Wan JN and Zhong Y. MiR-96-5p promotes the proliferation, invasion and metastasis of papillary thyroid carcinoma through down-regulating CCDC67. Eur Rev Med Pharmacol Sci 23: 3421-3430, 2019.

(i) (2) This work is licensed under a Creative Commons Attribution-NonCommercial-NoDerivatives 4.0 International (CC BY-NC-ND 4.0) License. 